\title{
Vertebrogenic dysphagia and gait disturbance mimicking motor neuron disease
}

A 60 year old man was admitted with a 1-2 year history of increasing dysphagia causing weight loss and a $5-10$ year history of progressive gait disturbance with stiffness and muscle twitches in both legs. Urgency micturition had recently developed, which was ascribed to the patient's known hypertrophy of the prostate. Except for prostatism and lumbago, his medical history was unremarkable. Although the family history was incomplete, it was known that the patient's father and three siblings all had developed difficulty with walking in midlife. Clinical examination showed dysphagia but otherwise the cranial nerves were intact. The deep tendon reflexes in the upper limbs were normal but were very brisk in the lower limbs, and pathological Babinski's reflexes were noted bilaterally. A familial motor neuron disease was suspected but EMG and motor evoked potentials of all four limbs were essentially normal. Spine MRI showed a posterior disc herniation at the C5/6 level with ventral compression of the cervical medulla and to the left. Also, the left root sleeve C6 was compressed. There was no intramedullar signal abnormality. Furthermore, the disc C4/5 showed a large anterior disc herniation with compression of the oesophagus and hypopharynx. An MRI of the thoracic and lumbosacral spine showed small insignificant disc herniations at the Th8/9 and L3/4 levels (figs 1, 2, and 3). The patient underwent successful discectomy. Three years later the patient remains symptom free and has returned to work.
Forestier-Rotes Querol syndrome (ankylosing hyperostosis of the spine) is a well established cause of dysphagia, but the present case does not fulfill the criteria for this syndrome, ${ }^{1}$ and his symptoms can solely be explained by the combined effect of the two large cervical disc herniations.

Cervical disc herniation is a well recognised differential diagnosis in early motor neuron disease usually associated with symptoms and signs from the upper limbs only. This particular patient developed severe dysphagia as well as symptoms from the lower limbs and sparing the upper limbs. This is the fourth case of symptomatic anterior cervical disc herniation to be reported. ${ }^{2}$

PETER M ANDERSEN Department of Neurology, Umeå University Hospital, S-901 85 Umeå, Sweden

Department of Neuroradiology

MARKKU FAGERLUND

Correspondence to: Dr P M Andersen

Peter.Andersen@neuro.umu.se

1 Marks B, Schober E, Swoboda H. Diffuse idiopathic skeletal hyperostosis causing obstructing laryngeal edema. Eur Arch Otorhinolaryngol $1998 ; 255: 256-8$

2 Bernardo KL, Grubb RL, Coxe WS, et al. Anterior cervical disc herniation. Case report. $\mathcal{F}$ Neurosurg 1988;69:134-6.
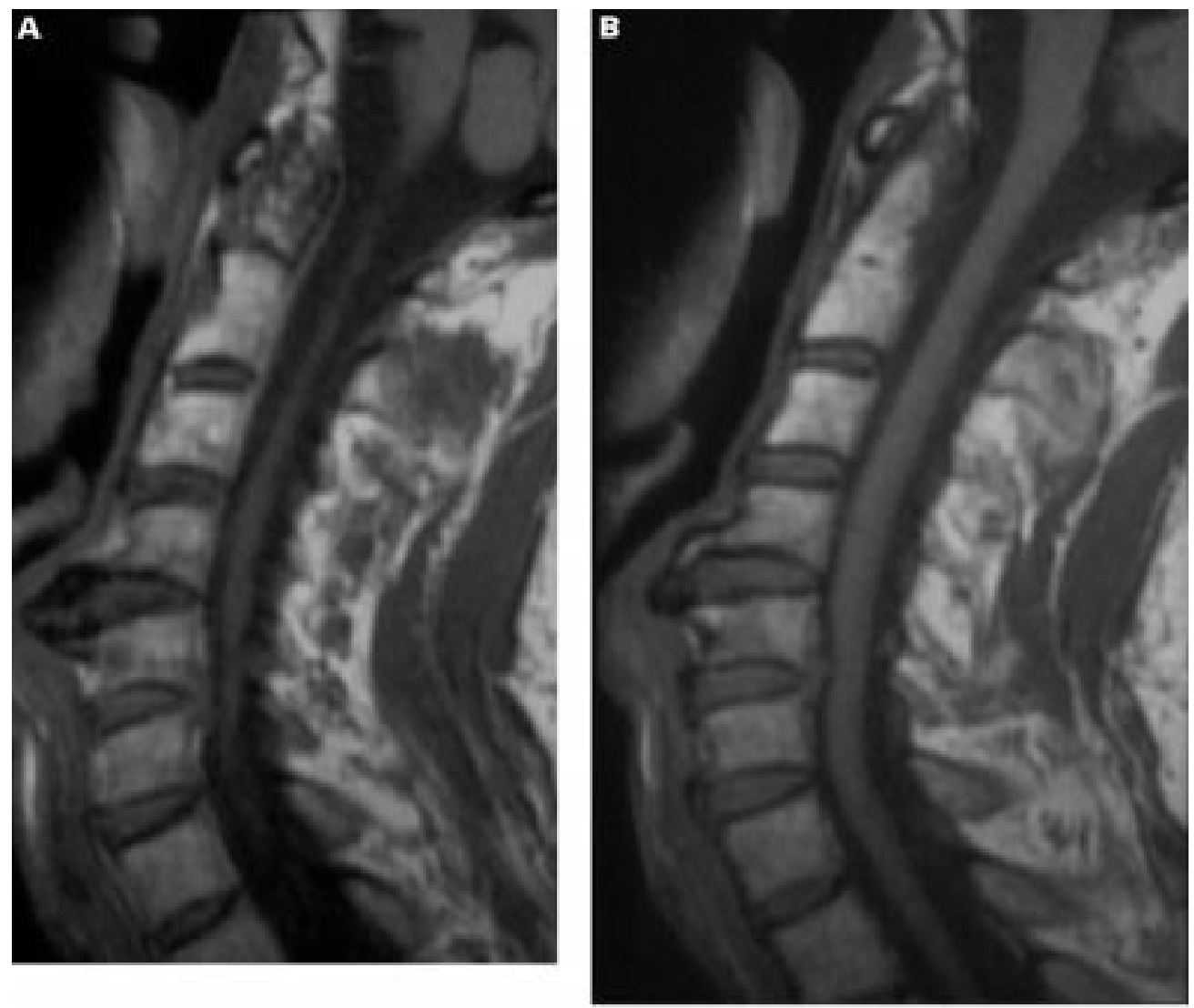

Figure 1 ( $A$ and $B) T 1$ weighted sagittal view. Large anterior $C 4 / 5$ disc herniations with broadened prevertebral soft tissue and compression of both oesophagus and larynx. Left sided disc herniation C5/6 with medullary compression. 

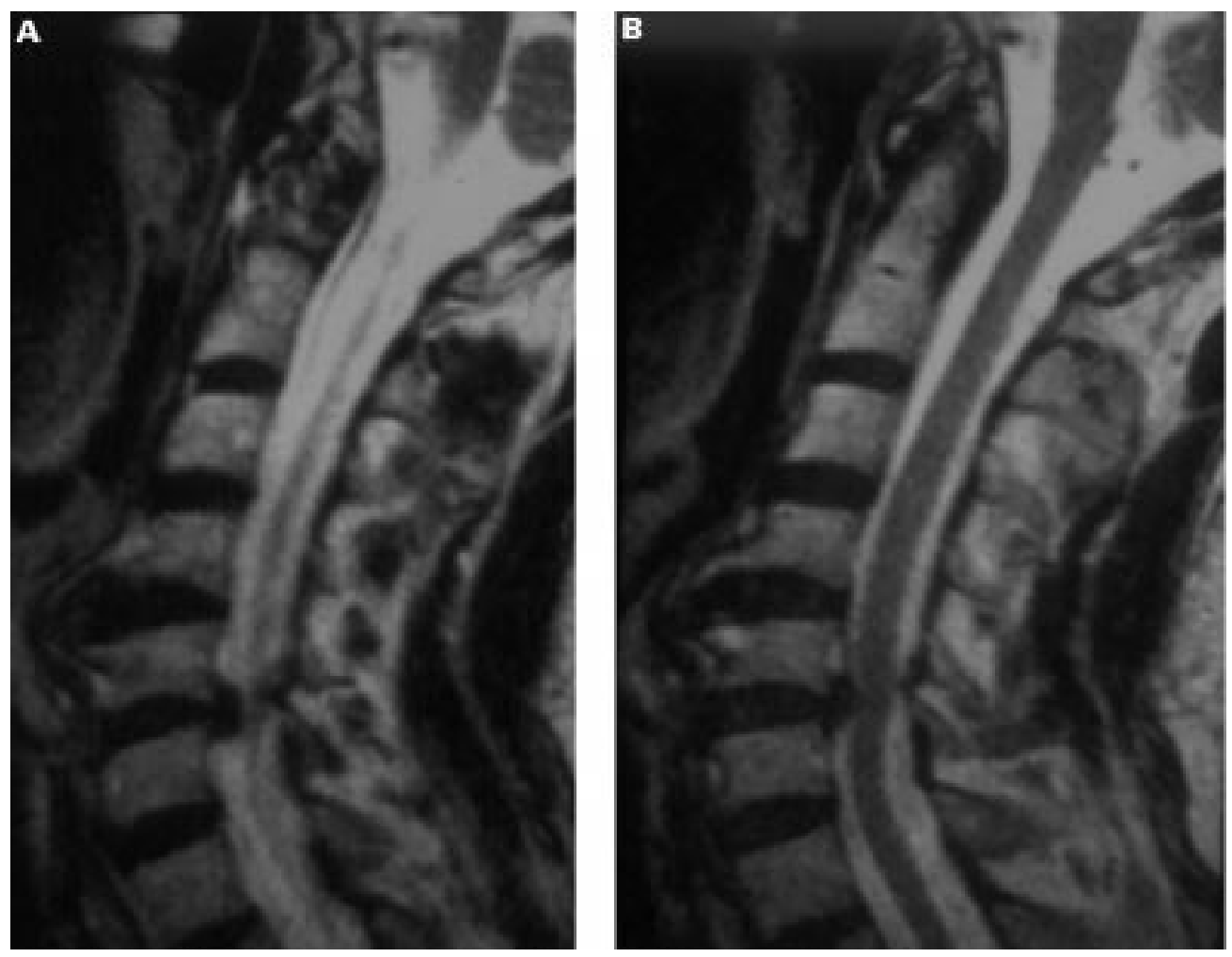

Figure 2 ( $A$ and B)T2 weighted sagittal view. The same discs show more clearly the medullary compression at the C5/6 level.
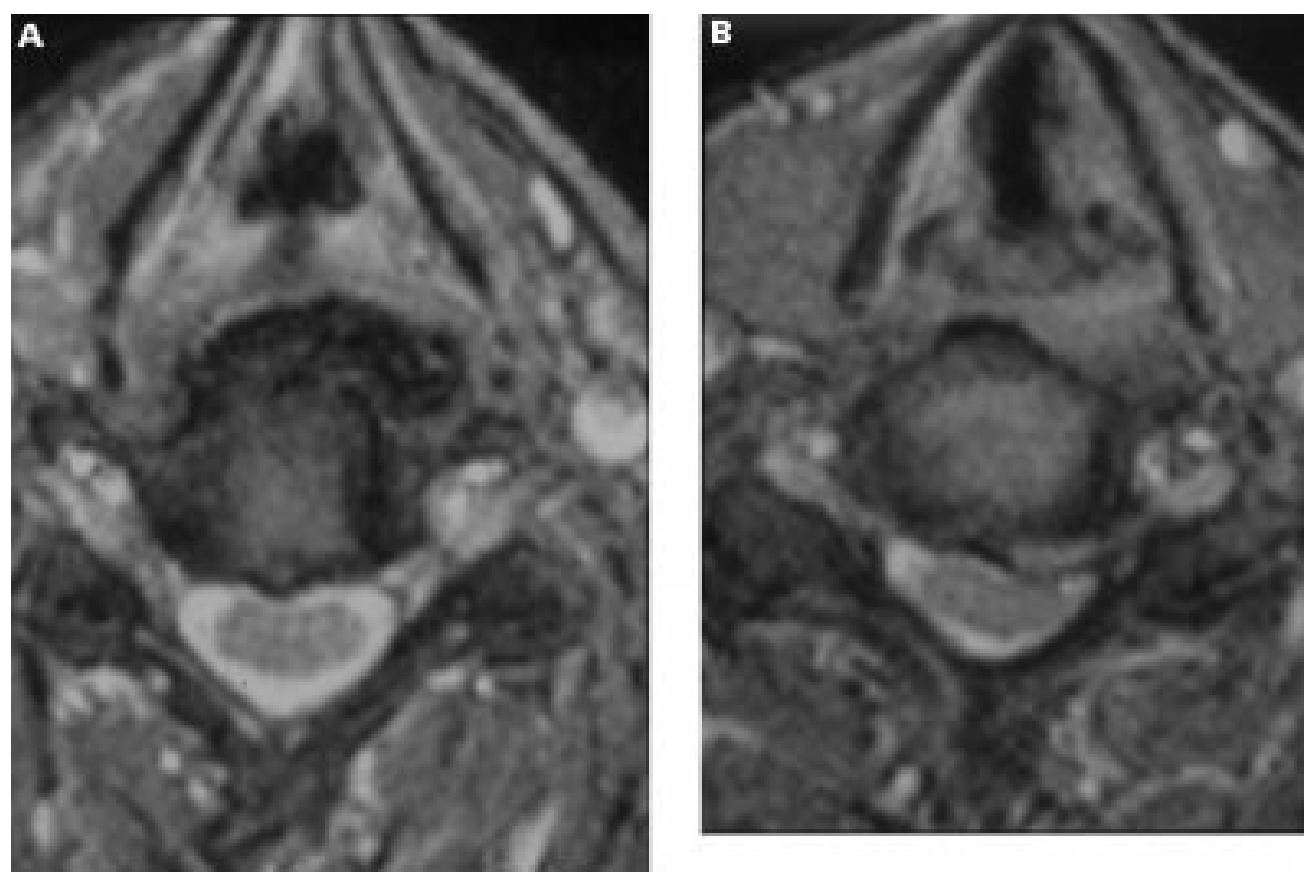

Figure 3 (A) Transverse T1 weighted image at the C4/5 level. Anterior extension of the large disc herniation. (B) Disc herniation at the C5/6 level showing the compression of the medulla. 